23. T. F. Heinz, in Nonlinear Surface Electromagnetic Phenomena, H.-E. Ponath, G. I. Stegeman, Eds. (Elsevier, Amsterdam, 1991), pp. 353-416.

24. J. M. Fraser, A. I. Shkrebtii, J. E. Sipe, H. M. van Driel, Phys. Rev. Lett. 83, 4192 (1999).

25. P. M. Echenique et al., Surf. Sci. Rep. 52, 219 (2004).

26. See supporting material on Science Online.

27. C. Reuß et al., Phys. Rev. Lett. 82, 153 (1999).
28. M. Roth, M. T. Pickel, ]. X. Wang, M. Weinelt, T. Fauster, Phys. Rev. Lett. 88, 096802 (2002).

29. K. Boger, M. Weinelt, T. Fauster, Phys. Rev. Lett. 92, 126803 (2004).

30. F. E. Olsson et al., Phys. Rev. B 70, 205417 (2004)

31. ]. Güdde, M. Rohleder, U. Höfer, Appl. Phys. A 85, 345 (2006).

32. ]. Shah, Ultrafast Spectroscopy of Semiconductors and Semiconductor Nanostructures (Springer-Verlag, Berlin, 1996).
33. Supported by Deutsche Forschungsgemeinschaft grant HO 2295/3 and by the Center for Optodynamics, Marburg, Germany.

Supporting Online Material

www.sciencemag.org/cgi/content/full/318/5854/1287/DC1

SOM Text

References

20 June 2007; accepted 3 October 2007

$10.1126 /$ science. 1146764

\title{
Emission of Coherent THz Radiation from Superconductors
}

\author{
L. Ozyuzer, ${ }^{1,2}$ A. E. Koshelev ${ }^{2}$ C. Kurter, ${ }^{2,3}$ N. Gopalsami, ${ }^{4}$ Q. Li, ${ }^{2}$ M. Tachiki, ${ }^{5}$ K. Kadowaki, ${ }^{6}$ \\ T. Yamamoto, ${ }^{6}$ H. Minami, ${ }^{6}$ H. Yamaguchi, ${ }^{6}$ T. Tachiki, ${ }^{7}$ K. E. Gray, ${ }^{2}$ W.-K. Kwok, ${ }^{2}$ U. Welp ${ }^{2 *}$
}

Compact solid-state sources of terahertz $(\mathrm{THz})$ radiation are being sought for sensing, imaging, and spectroscopy applications across the physical and biological sciences. We demonstrate that coherent continuous-wave $\mathrm{THz}$ radiation of sizable power can be extracted from intrinsic josephson junctions in the layered high-temperature superconductor $\mathrm{Bi}_{2} \mathrm{Sr}_{2} \mathrm{CaCu}_{2} \mathrm{O}_{8}$. In analogy to a laser cavity, the excitation of an electromagnetic cavity resonance inside the sample generates a macroscopic coherent state in which a large number of junctions are synchronized to oscillate in phase. The emission power is found to increase as the square of the number of junctions reaching values of 0.5 microwatt at frequencies up to $0.85 \mathrm{THz}$, and persists up to $\sim 50$ kelvin. These results should stimulate the development of superconducting compact sources of $\mathrm{THz}$ radiation.

$\mathrm{T}$ he observed gap in the generation of electromagnetic radiation, extending approximately from $0.5 \mathrm{THz}$ to $2 \mathrm{THz}$, stems from the separation of the two general paradigms for generating electromagnetic waves (1-3): alternating currents in semiconductorbased electronics and electronic transitions between quantized electronic states in lasers, respectively. The frequency of semiconductor devices is bounded from above by limits of the electron velocities, whereas the frequency of solidstate lasers is bounded from below by thermal energies that limit the smallest electronic transitions useful for lasing. Josephson junctionstwo superconducting electrodes separated by a thin insulating layer-naturally convert dc voltages into high-frequency electromagnetic radiation spanning the $\mathrm{THz}$ gap, with $1 \mathrm{mV}$ corresponding to $0.483 \mathrm{THz}$. Although the emission from a single junction is weak, many such junctions emitting in phase at the same frequency can produce useful emission power (4-6). Stacks

${ }^{1}$ Department of Physics, Izmir Institute of Technology, TR35430 Izmir, Turkey. ${ }^{2}$ Materials Science Division, Argonne National Laboratory, Argonne, IL 60439, USA. ${ }^{3}$ Physics Division, Illinois Institute of Technology, Chicago, IL 60616, USA. ${ }^{4}$ Nuclear Engineering Division, Argonne National Laboratory, Argonne, IL 60439, USA. ${ }^{5}$ Graduate School of Frontier Sciences, The University of Tokyo, 5-1-5 Kashiwanoha, Kashiwa 277-8568, Japan. ${ }^{6}$ Institute of Materials Science, University of Tsukuba, 1-1-1 Tennodai, Tsukuba-shi, Ibaraki-ken 305-8577, Japan. ${ }^{7}$ Department of Electrical and Electronic Engineering, National Defence Academy, Hashirimizu 1-10-20, Yokosuka, Kanagawa 2398686, Japan.

*To whom correspondence should be addressed. E-mail: welp@anl.gov of junctions with unsurpassed packing density occur naturally in the layered high-temperature superconductor $\mathrm{Bi}_{2} \mathrm{Sr}_{2} \mathrm{CaCu}_{2} \mathrm{O}_{8}$ (BSCCO). This material, composed of superconducting $\mathrm{CuO}_{2}$ layers that are coupled through the intrinsic Josephson effect (7), can sustain high voltages across the junctions and holds the potential for very intense, coherent radiation $(8-10)$ that covers the $\mathrm{THz}$ gap. However, the key requirement for producing useful radiation from BSCCO, namely achieving synchronization of the high-frequency oscillations of all the junctions in the stack, has so far been a major challenge preventing the realization of this potential. Various approaches for synchronizing the junctions have been considered, such as applying a magnetic field to induce coherent Josephson vortex flow (11-16) or inserting the BSCCO crystal into a microwave cavity (17). However, the far-field radiation power obtained from BSCCO is limited to the $\mathrm{pW}$ range (18).

We show that $\mathrm{THz}$ radiation power in the $\mu \mathrm{W}$ range can be produced using a method by which the phase of the emission from the atomic scale Josephson junctions in BSSCO is synchronized by a standing electromagnetic wave that is formed by multiple reflections in the cavity formed by the side surfaces of the crystal, exactly as in a laser. Electromagnetic waves inside a BSCCO crystal propagate as Josephson plasma modes (19-21), which resemble the guided modes in an optical waveguide. The average electric field on the side surfaces cancels in all but the in-phase mode, so only this mode produces noticeable emission (Fig. 1, C and D). Resonances that occur on the long dimension of the mesa incur sign changes of the electric field on the long side faces and do not contribute to the emission in a substantial way. In the in-phase mode, the coherent superposition of the electromagnetic waves from each junction creates a macroscopic coherent state in which the radiation power increases as the square of the number of junctions. We report that more than 500 junctions can be made to oscillate in phase, producing continuous wave coherent radiation power up to $\sim 0.5 \mu \mathrm{W}$ at frequencies up to $0.85 \mathrm{THz}$. The available power is potentially much larger, because there is evidence that $20 \mu \mathrm{W}$ of power are pumped into the observed $\mathrm{THz}$ cavity resonance. The emission persists up to temperatures of $\sim 50 \mathrm{~K}$. In contrast to previous studies (11-16), emission does not require the application of a magnetic field, considerably simplifying the design of superconducting $\mathrm{THz}$ sources. In fact, a single applied dc current leads to the efficient excitation of continuous coherent $\mathrm{THz}$ radiation.

We report results on a series of BSCCO samples in the form of mesas (Fig. 1, A and B) with widths varying from $40 \mu \mathrm{m}$ to $100 \mu \mathrm{m}$, a length of $300 \mu \mathrm{m}$, and a height of about $1 \mu \mathrm{m}$. The mesas and the electrical contacts are fabricated in a series of thermal evaporation, photolithography, and Ar ion milling steps (22).

Figure 2 shows the current-voltagecharacteristics (IV-c) and the radiation power detected by the bolometer. Both quantities are recorded simultaneously as a function of decreasing bias voltage for the parallel and perpendicular settings of a parallel-plate cut-off filter (22). The data reveal peaks of radiation near $0.37 \mathrm{~V}$ and $0.71 \mathrm{~V}$ that are polarized with their E-field perpendicular to the $\mathrm{CuO}_{2}$-planes, and unpolarized radiation at high current and voltage bias. The former is identified as Josephson radiation, whereas the latter is thermal radiation. Upon rotating the cut-off filter, both peaks decrease in the same proportion, indicating the same radiation frequency. The peak power in Fig. 2 is around $11 \mathrm{nW}$, and we recorded up to $50 \mathrm{nW}$ when no filters are inserted into the beam path (fig. S4). Correcting for the collection angle of the bolometer yields a total radiation power of the sample of $\sim 0.5 \mu \mathrm{W}$. These values are more than $10^{4}$ times as large as previous reports on far-field radiation from BSCCO mesas (18). The backbending of the IV-c and the unpolarized thermal radiation indicate heating of the mesa at the highest currents. The effective mesa temperatures along the IV-c, shown in Fig. 2, are estimated by 
equating (23) the mesa resistivity to the quasiparticle resistivity, $\rho_{\mathrm{c}}(\mathrm{T})$, of BSCCO (fig. S5). Also included in Fig. 2 is a simulation of the thermal radiation obtained with the help of the Stefan-Boltzmann law and a numerical solution of the heat-diffusion equation (22). The agreement of the simulation with the data shows that our thermal model is accurate and that thermal management in such large mesas can be achieved.

An estimation of the radiation frequency is given by the filter cut-off frequency at which the polarization ratio levels off (Fig. $3 \mathrm{~A}$ ) and by far-infrared spectra (Fig. 3B). Both methods yield consistent results, i.e., 0.36, 0.48, 0.59, and $0.85 \mathrm{THz}$ for the $100-\mu \mathrm{m}, 80-\mu \mathrm{m}, 60-\mu \mathrm{m}$ and $40-\mu \mathrm{m}$ wide mesas, respectively. The value of $0.85 \mathrm{THz}$ is the highest frequency of far-field radiation from a superconducting source yet observed (fig. S8). These frequency values are in good agreement with the fundamental cavity resonance, $f=c_{0} / 2 n w$, where $w$ is the width of the mesa and $n \approx 3.5$ is the $c$-axis far-infrared refractive index of BSCCO (24), which for $w=$ $80 \mu \mathrm{m}$ yields $f=0.52 \mathrm{THz}$. Furthermore, the observed radiation frequencies increase approximately proportionally to $1 / w$ (see inset of Fig. 3 B), demonstrating that they correspond to a cavity resonance. To excite this resonance, the Josephson frequency, $f=V_{j c t} / \Phi_{0}$ should match the reso- nance frequency, where $\Phi_{0}$ is the flux quantum and $V_{j c t}$ the voltage per junction. Using the mesa height of $1.1 \mu \mathrm{m}$ as determined from atomic force microscopy, and $1.56 \mathrm{~nm}$ spacing of the $\mathrm{CuO}_{2}$-double layers, the voltage shown in Fig. 2 for the large radiation peak of the $80-\mu \mathrm{m}$ mesa corresponds to $0.49 \mathrm{THz}$, consistent with the spectroscopic data. Thus, upon decreasing the bias from the fully resistive state, the emission power builds up as the Josephson frequency comes into resonance with the cavity. With decreasing voltage, some junctions may switch back from the resistive into the superconducting state (25). During such a jump the voltage per remaining resistive junction and the Josephson frequency increase. Consequently, the system falls out of resonance with the cavity mode, and emission ceases. With further decreasing bias, the resonance is again approached from above, and the behavior repeats itself, yielding a second emission peak involving a smaller number of active junctions.

Radiation from the same sample containing a varying number of emitting junctions allows for a direct test of coherency. Figure 4A displays a sequence of emission peaks for positive and negative bias voltages on the $80-\mu \mathrm{m}$ mesa. The fraction of active junctions, $n_{\text {rel }}$, relative to the highest emission peak, can be determined directly from the resistances corresponding to the
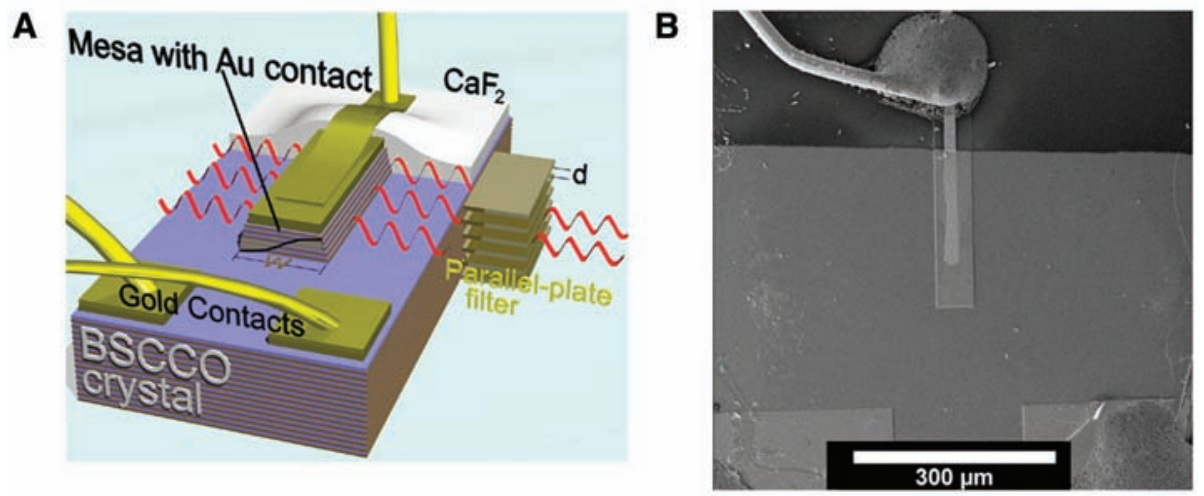

C

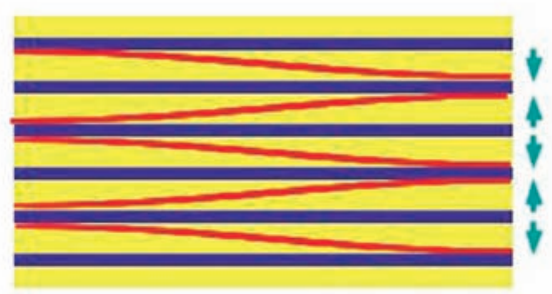

D

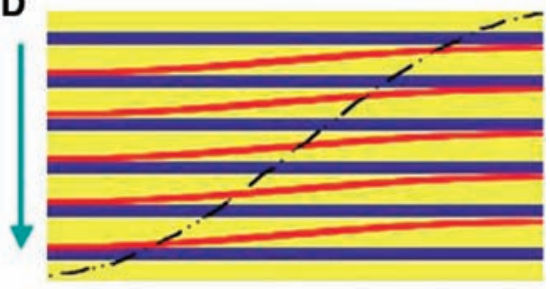

Fig. 1. (A) Schematic of the BSCCO mesas. The applied c-axis current excites the fundamental cavity mode (solid half-wave) on the width $w$ of the mesa, and high-frequency electromagnetic radiation is emitted from the side faces (red waves), whose polarization and frequency are analyzed with parallel-plate filters (22). (B) Scanning electron microscopy image of the mesa. Schematics of the anti-phase (C) and of the in-phase (D) mode. The blue and yellow layers are the $\mathrm{CuO}_{2}$ layers and $\mathrm{Bi}-\mathrm{Sr}-\mathrm{O}$ layers. The red waves represent the alternating electric field. In the anti-phase mode the electric field on the long side face largely cancels, resulting in negligible emission. For the in-phase mode, the electric fields from each junction add to create an intense standing wave (black dashed line) and strong emission.

various branches in the IV-c (fig. S7). An analysis $(10,26)$ of the loss mechanisms shows that for mesas with height less than 1.0 to $1.5 \mu \mathrm{m}$, quasiparticle losses are larger than losses due to radiation. In this limit, the emitted power increases like the square of the number of coherently oscillating junctions. The experimentally observed peak powers are proportional to $n_{\text {rel }}^{2}$, demonstrating directly that the junctions in the stack emit coherently. Figure 4C shows a closeup of the return branch of IV-c and of the radiation power of the third peak in Fig. 4A. The absence of a jump in the IV-c allows us to establish a baseline of the current and to determine the excess current that supplies the energy for the excitation of the cavity resonance. The height of the resonance in the IV-c depends on the quality factor of the cavity resonance (26). These data suggest that about $20 \mu \mathrm{W}$-about $2.5 \%$ of the total dc power dissipated in the mesaare pumped into the in-phase resonance, implying that considerably enhanced radiation powers could be obtained with this mesa, for example, through improved impedance matching with the help of antennas, gratings, or dielectric coatings.

In a homogeneous mesa in zero magnetic field, the Josephson oscillations do not couple directly to the cavity resonances. However, when the Josephson frequency is twice as large as the cavity frequency, the resonance may be excited as a result of a parametric instability leading to so-called zero field steps in the IV-c, which were observed in conventional Josephson junctions (27). Our frequency analysis rules out this excitation mechanism. A plausible mechanism of coupling can arise from an asymmetric modulation of the Josephson critical current

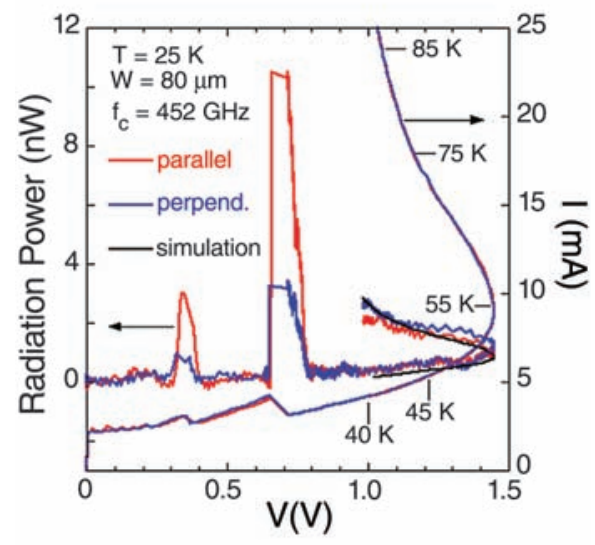

Fig. 2. Current-voltage characteristics and radiation power of the $80-\mu \mathrm{m}$ mesa. The voltage dependence of the current (right $y$-axis) and of the radiation power (left $y$-axis) at $25 \mathrm{~K}$ for parallel and perpendicular settings of the filter with 0.452 $\mathrm{THz}$ cut-off frequency are shown for decreasing bias in zero applied magnetic field. Polarized Josephson emission occurs near 0.71 and $0.37 \mathrm{~V}$, and unpolarized thermal radiation occurs at higher bias. The black solid line is a simulation of the thermal radiation (22). 


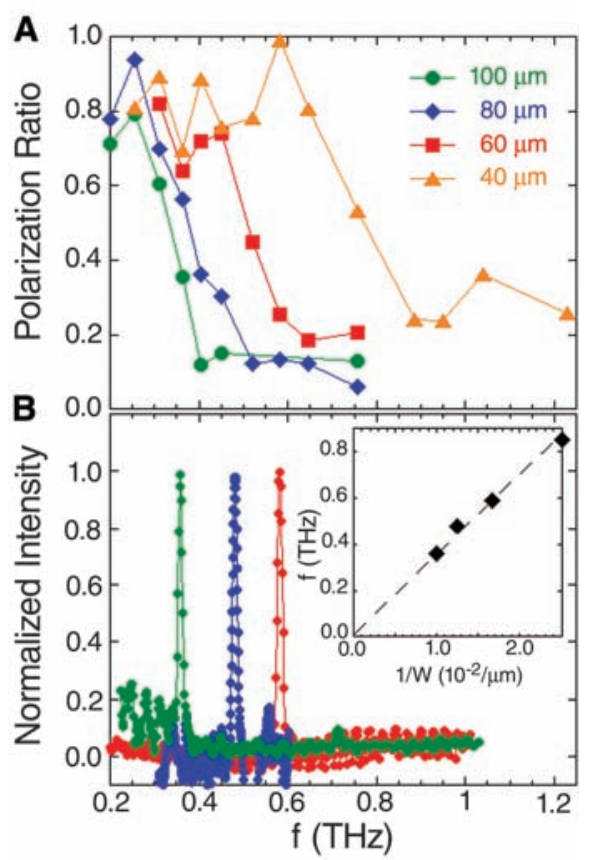

Fig. 3. Spectral characterization of the emission. (A) The polarization ratio - defined as the ratio the radiation power measured at perpendicular and at parallel filter settings - of the emission peaks is shown for four mesas as a function of cut-off frequency of the filters. The radiation frequency is estimated from the filter cut-off frequency at which the polarization ratio levels off at high frequencies (22). (B) Far-infrared spectra of the Josephson radiation. Sharp emission lines are clearly resolved. The observed line width of $\sim 9 \mathrm{GHz}$ (FWHM) is instrument-resolution limited. The scaling of the emission frequency with the inverse mesa width, shown in the inset, demonstrates that a cavity resonance on the width is excited.

along the width of the mesa, due to, for example, an inhomogeneous oxygen concentration or defects induced during fabrication processes. For single junctions, the coupling of a modulated critical current distribution to cavity resonances has been observed (28). A recent quantitative analysis of radiation and transport properties of mesas with such modulation (26) suggests that the radiation power from the mesas described here can be as high as $1 \mathrm{~mW}$ under optimized conditions. Therefore, we anticipate that the observed radiation power can be significantly increased by enhancing the coupling to the internal cavity mode by deliberately introduced strong modulations of the Josephson critical current. There is a large effort to develop quantum cascade lasers as sources of $\mathrm{THz}$ radiation (1). Presently, their lowest emission frequency is $\sim 1.6 \mathrm{THz}$, with a continuous wave emission power of $\sim 0.5 \mathrm{~mW}(1,29)$. The superconducting sources described here are a competitive technology for the frequency range of $0.5 \mathrm{THz}$ to $1.5 \mathrm{THz}$, which is also the range where compact solid-state sources are most lacking.
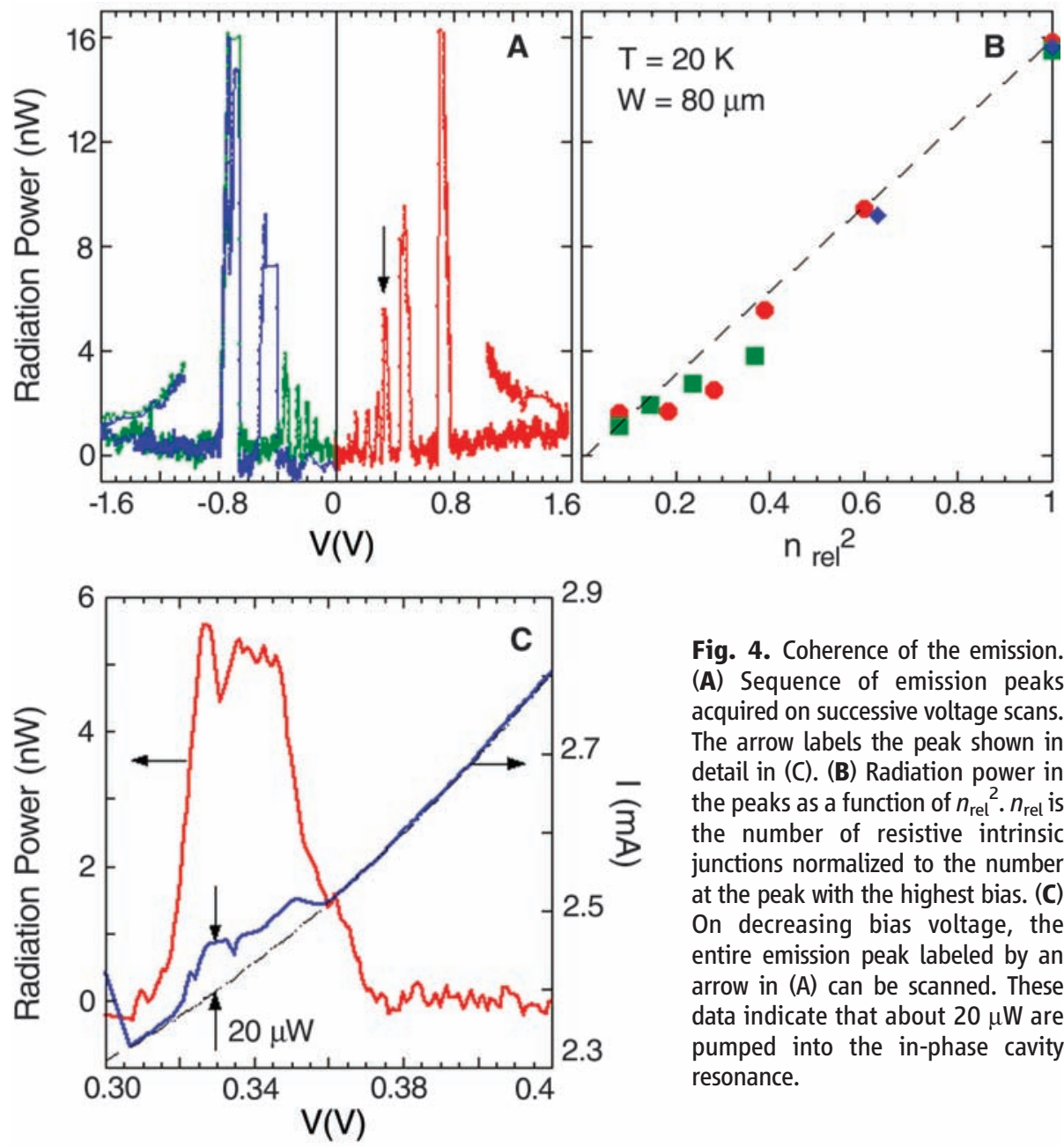

Fig. 4. Coherence of the emission. (A) Sequence of emission peaks acquired on successive voltage scans. The arrow labels the peak shown in detail in (C). (B) Radiation power in the peaks as a function of $n_{\text {rel }}{ }^{2} \cdot n_{\text {rel }}$ is the number of resistive intrinsic junctions normalized to the number at the peak with the highest bias. (C) On decreasing bias voltage, the entire emission peak labeled by an arrow in (A) can be scanned. These data indicate that about $20 \mu \mathrm{W}$ are pumped into the in-phase cavity resonance.

\section{References and Notes}

1. M. Tonouchi, Nat. Photon. 1, 97 (2007).

2. M. Lee, M. C. Wanke, Science 316, 64 (2007).

3. A. Borak, Science 308, 638 (2005).

4. M. Darula, T. Doderer, S. Beuven, Supercond. Sci. Technol. 12, R1 (1999).

5. A. K. Jain, K. K. Likharev, J. E. Lukens, J. E. Sauvageau, Phys. Rep. 109, 309 (1984).

6. P. Barbara, A. B. Cawthorne, S. V. Shitov, C. J. Lobb, Phys. Rev. Lett. 82, 1963 (1999).

7. R. Kleiner, F. Steinmeyer, G. Kunkel, P. Müller, Phys. Rev. Lett. 68, 2394 (1992).

8. T. Koyama, M. Tachiki, Solid State Commun. 96, 367 (1995).

9. M. Tachiki, M. lizuka, K. Minami, S. Tejima, H. Nakamura, Phys. Rev. B 71, 134515 (2005).

10. L. N. Bulaevskii, A. E. Koshelev, J. Superconduct. Novel Magnetism 19, 349 (2006).

11. H. B. Wang et al., Appl. Phys. Lett. 89, 252506 (2006).

12. M.-H. Bae, H.-J. Lee, ].-H. Choi, Phys. Rev. Lett. 98, 027002 (2007).

13. K. Kadowaki et al., Physica C 437-438, 111 (2006).

14. A. Irie, Y. Hirai, G. Oya, Appl. Phys. Lett. 72, 2159 (1998).

15. V. M. Krasnov, N. Mros, A. Yurgens, D. Winkler, Phys. Rev. B 59, 8463 (1999).

16. S. M. Kim et al., Phys. Rev. B 72, 140504 (2005).

17. S. Madsen, G. Fillatrella, N. F. Pedersen, Eur. Phys. J. B 40, 209 (2004).

18. I. E. Batov et al., Appl. Phys. Lett. 88, 262504 (2006).

19. R. Kleiner, Phys. Rev. B 50, 6919 (1994).

20. L. N. Bulaevskii, M. Zamora, D. Baeriswyl, H. Beck, J. R. Clem, Phys. Rev. B 50, 12831 (1994)

21. N. F. Pedersen, S. Sakai, Phys. Rev. B 58, 2820 (1998).

22. Materials and methods are available as supporting material on Science Online.
23. A. Yurgens, D. Winkler, N. V. Zavaritsky, T. Claeson, Phys. Rev. Lett. 79, 5122 (1997)

24. M. B. Gaifullin, M. Matsuda, N. Chikumoto, J. Shimoyama, K. Kishio, Phys. Rev. Lett. 84, 2945 (2000).

25. M. Machida, T. Koyama, M. Tachiki, Phys. Rev. Lett. 83, 4618 (1999).

26. A. E. Koshelev, L. N. Bulaevskii, cond-mat/0708.3269.

27. T. A. Fulton, R. C. Dynes, Solid State Commun. 12, 57 (1973)

28. M. Russo, R. Vaglio, Phys. Rev. B 17, 2171 (1978).

29. C. Walther, G. Scalari, J. Faist, H. Beere, D. Ritchie, Appl. Phys. Lett. 89, 231121 (2006).

30. We thank L. Bulaevskii for many illuminating discussions; A. Imre for assistance with the microscopy performed at the Electron Microscopy Center and at the Center for Nanoscale Materials, both at Argonne National Laboratory; and R. Eyes for quantitative analysis of the transmission through parallel-plate filters. This work was supported by the U.S. Department of Energy-Basic Energy Sciences under contract DE-AC02-06CH11357; the Japanese Ministry of Education, Culture, Sports, Science, and Technology; and the Turkish TUBITAK under project 106T053. We thank the Institute for Theoretical Sciences, a joint institute of Argonne National Laboratory and the University of Notre Dame, for program coordination support.

Supporting Online Material

www.sciencemag.org/cgi/content/full/318/5854/1291/DC1 Materials and Methods

Figs. S1 to S8

References

28 August 2007; accepted 2 October 2007 10.1126/science. 1149802 


\section{Science MIAAAS}

Emission of Coherent THz Radiation from Superconductors L. Ozyuzer, A. E. Koshelev, C. Kurter, N. Gopalsami, Q. Li, M. Tachiki, K. Kadowaki, T. Yamamoto, H. Minami, H. Yamaguchi, T. Tachiki, K. E. Gray, W.-K. Kwok and U. Welp (November 23, 2007)

Science 318 (5854), 1291-1293. [doi: 10.1126/science.1149802]

Editor's Summary

This copy is for your personal, non-commercial use only.

Article Tools Visit the online version of this article to access the personalization and article tools:

http://science.sciencemag.org/content/318/5854/1291

Permissions Obtain information about reproducing this article:

http://www.sciencemag.org/about/permissions.dtl

Science (print ISSN 0036-8075; online ISSN 1095-9203) is published weekly, except the last week in December, by the American Association for the Advancement of Science, 1200 New York Avenue NW, Washington, DC 20005. Copyright 2016 by the American Association for the Advancement of Science; all rights reserved. The title Science is a registered trademark of AAAS. 